\title{
Indirect Treatment Comparison of Inotuzumab Ozogamicin Versus Blinatumomab for Relapsed or Refractory Acute Lymphoblastic Leukemia
}

\author{
Irina Proskorovsky • Yun Su • Kyle Fahrbach · Erik Vandendries • \\ Véronique Pagé · Uchenna Onyekwere · Yunyang Wang · \\ Joseph C. Cappelleri · Matthias Stelljes
}

Received: April 3, 2019 / Published online: May 28, 2019

(C) The Author(s) 2019

\begin{abstract}
Introduction: No head-to-head studies have compared inotuzumab ozogamicin ( $\mathrm{InO}$ ) and blinatumomab (Blina) for the treatment of adults with relapsed or refractory B cell precursor acute lymphoblastic leukemia (ALL). Indirect treatment comparisons (ITCs), namely network meta-analysis (NMA), anchored
\end{abstract}

Enhanced Digital Features To view enhanced digital features for this article go to https://doi.org/10.6084/ m9.figshare.8109149.

Electronic supplementary material The online version of this article (https://doi.org/10.1007/s12325019-00991-w) contains supplementary material, which is available to authorized users.

I. Proskorovsky $(\varangle) \cdot$ V. Pagé · Y. Wang

Evidera, St-Laurent, QC, Canada

e-mail: irina.proskorovsky@evidera.com

Y. Su

Pfizer Inc, New York, NY, USA

K. Fahrbach · U. Onyekwere

Evidera, Waltham, MA, USA

E. Vandendries

Pfizer Inc, Cambridge, MA, USA

J. C. Cappelleri

Pfizer Inc, Groton, CT, USA

M. Stelljes

University Hospital, Münster, Germany matching-adjusted indirect comparison (MAIC), and simulated treatment comparison (STC), were conducted to compare the relative efficacy of these therapies.

Methods: Patient-level data from a study that evaluated InO with standard of care (SoC) chemotherapy (INO-VATE-ALL) and published data from a study that evaluated Blina with SoC chemotherapy (TOWER) were used in the analyses. Endpoints evaluated included remission rate defined as complete remission or complete remission with incomplete hematologic recovery (CR/CRi), hematopoietic stem cell transplantation (HSCT), overall survival (OS), and event-free survival (EFS). For each outcome, treatment-effect modifiers were adjusted for in the anchored MAIC and STC analyses.

Results: Analyses showed statistically significant higher rates of remission and HSCT with InO compared to Blina irrespective of the ITC method used or measure of the effect (i.e., odds ratio $[\mathrm{OR}]$ or rate difference). The treatment effects derived from the MAIC and STC analyses were consistent and stronger than those estimated from the NMA. A trend favoring InO was detected for EFS. The ITC results for OS suggest no difference between InO and Blina.

Conclusion: Results from these ITCs indicated a statistically significant advantage for InO over Blina for rates of remission and HSCT in adults with relapsed or refractory B cell precursor ALL. It was not possible to fully adjust for all treatment-effect modifiers, and the similarity in 
chemotherapy regimens used in the SoC comparator arms of the INO-VATE-ALL and TOWER studies is worthy of further exploration. Both studies, however, used chemotherapy regimens that have a low response rate; therefore, no significant differences in efficacy outcomes are expected between SoC arms.

Funding: Pfizer Inc, New York, NY.

Plain Language Summary: Plain language summary available for this article.

Keywords: Blinatumomab; Indirect treatment comparison; Inotuzumab ozogamicin; Matching-adjusted indirect comparison; Relapsed or refractory acute lymphoblastic leukemia; Simulated treatment comparison

\section{PLAIN LANGUAGE SUMMARY}

Acute lymphoblastic leukemia (ALL) is a rare blood cancer typically diagnosed in children or adults over 50, with a high rate of recurrence. While initial chemotherapy regimens can bring about complete remission in high proportions of adults with ALL, the disease will recur in many; this is called "relapsed" or "refractory" (R/R) ALL. With each recurrence, treatments become less effective. The best long-term survival option for patients in remission is transplantation of the stem cells that produce our blood components (hematopoietic stem cell transplantation, or HSCT). But not everyone can tolerate HSCT, particularly older, frailer patients, and ALL can recur yet again. The 5-year survival among patients with R/R ALL is only $10 \%$.

Two immunotherapy drugs were US-approved for second-line or later treatment of $\mathrm{R} / \mathrm{R}$ ALL, inotuzumab ozogamicin (InO) and blinatumomab (Blina). Their individual efficacy was demonstrated in clinical trials, but understanding their comparative efficacy is important for medical and economic decision-makers. Since no direct comparison of the two via clinical trial has been done yet, we used indirect treatment comparison (ITC) methods to assess their relative efficacy.

We conducted several types of ITCs (network meta-analyses [NMA], matching-adjusted indirect comparisons [MAIC], and simulated treatment comparisons [STC]), using data from the two clinical trials, INO-VATE-ALL for InO and TOWER for Blina. The ITC results indicated higher rates of remission and of HSCT for InO over Blina, a trend favoring InO for event-free survival (EFS), and no difference between them in overall survival (OS).

\section{INTRODUCTION}

Acute lymphoblastic leukemia (ALL) is a rare, heterogeneous, hematologic disease resulting from malignant transformation and proliferation of progenitor lymphoid cells $[1,2]$. The disease is characterized by an accumulation of lymphoblasts in the bone marrow, peripheral blood, and other organs [1-3]. In adults with ALL, B cell lineage represents approximately $75 \%$ of cases, with the remaining cases being $\mathrm{T}$ cell lineage [1]. Precursor B cell ALL is usually associated with the expression of CD10, CD19, CD22, CD34, and CD79a on the cell surface $[1,3]$.

In the USA, the age-adjusted incidence rate for ALL is 1.58 per 100,000 individuals per year [1]. For 2018, it was estimated that 5920 new cases were diagnosed and 1470 deaths due to the disease were observed in the USA [4]. Diagnosis of ALL generally occurs either during childhood or later in adulthood, after 50 years of age [2].

Although ALL is the most common form of pediatric acute leukemia, the disease accounts for $20 \%$ of leukemias in adults and is particularly devastating in this population $[1,2,5]$. In adults, approximately $80-90 \%$ of patients will achieve a complete response with initial therapy; however, most will eventually relapse, with worse outcomes observed in older adults [6]. After relapse, response rates decrease, particularly for patients whose first remission was short. The 5-year survival among patients with relapsed or refractory (R/R) ALL is only $10 \%$.

The foundation of treatment includes systemically administered combination chemotherapy [2]. Induction, consolidation, and outpatient maintenance comprise the treatment phases of ALL, with central nervous system prophylaxis administered during periods of each phase. The goal of induction therapy is to achieve complete remission, after which 
patients may undergo allogeneic hematopoietic stem cell transplantation (HSCT) or progress to the consolidation and maintenance phases. For adult patients with R/R ALL, HSCT offers the best option for long-term survival; however, prior to HSCT, a complete response to therapy is typically required, which is achieved by only approximately $40 \%$ of patients after the first salvage therapy $[2,6-8]$. The introduction of novel therapies including immunotherapies as salvage therapy have offered the potential for long-term survival in these patients. Immunotherapies include monoclonal antibodies, conjugated monoclonal antibodies, bispecific $\mathrm{T}$ cell engagers, and chimeric antigen receptor T cell therapies [6].

Inotuzumab ozogamicin (Pfizer, Philadelphia, PA, USA) and blinatumomab (Amgen, Thousand Oaks, CA, USA) are both approved for the treatment of adults with $\mathrm{R} / \mathrm{R} \mathrm{B}$ cell precursor ALL $[9,10]$. Blinatumomab (Blina), a bispecific T cell engager, binds CD19 expressed on the surface of B-lineage cells to CD3 on cytotoxic lymphocytes resulting in CD19-mediated cell death [6]. Inotuzumab ozogamicin (InO), a conjugate monoclonal antibody, is composed of a monoclonal antibody targeting CD22 covalently linked with the cytotoxic agent calicheamicin $[6,10]$. After binding to the CD22 antigen on B cells, the CD22-conjugate complex is internalized where the cytotoxic agent is released, thus leading to apoptosis. The efficacy of both Blina and InO were demonstrated in their respective phase III studies [11, 12].

Evidence that compares the efficacy of InO with that of Blina is important for clinical and economic decision-making. To date, there have been no direct comparative studies; therefore, indirect treatment comparisons (ITCs) are needed to assess the relative efficacy of these therapies. Network meta-analysis (NMA) is an ITC method that is commonly used to estimate relative treatment effects from clinical trials that have a common comparator and patient populations that are homogeneous [13, 14]. When the study populations involved in the comparisons are heterogeneous, as is the case with INOVATE-ALL (NCT01564784) and TOWER (NCT02013167), alternative methods can be used to adjust for imbalances in risk factors that are suspected treatment-effect modifiers before estimating relative treatment effects $[14,15]$. These methods include anchored matching-adjusted indirect comparison (MAIC) and simulated treatment comparison (STC), which have been recognized by health technology assessment authorities [16].

The primary objective of this analysis was to indirectly compare the efficacy of InO with that of Blina among adult patients with R/R ALL using data from the INO-VATE-ALL and TOWER studies. Outcomes evaluated were complete remission or complete remission with incomplete hematologic recovery (CR/CRi), HSCT, overall survival (OS), and event-free survival (EFS).

\section{METHODS}

\section{Data Sources}

The efficacies of InO and Blina in adult patients with R/R ALL were proven in two separate phase III, randomized, open-label studies [11, 12]. INO-VATE-ALL compared InO and standard of care (SoC) chemotherapy [12]. Blinatumomab was compared with SoC chemotherapy in the TOWER study $[11,17]$. The proposed ITCs used individual patient-level data from INO-VATEALL (cutoff date of January 4, 2017) and published summary data from TOWER.

No institutional board review was required for this study as it was based on a post hoc analysis of previously published data from the INO-VATE-ALL and TOWER trials. These previous studies involved human participants and were conducted in accordance with the ethical standards of the institutional and/or national research committees of each study's investigative sites, and with the 1964 Helsinki declaration and its later amendments, or comparable ethical standards. Informed consent was obtained from all individual participants included in these previous trials. 


\section{Study Compatibility Assessment}

Compatibility of the INO-VATE-ALL and TOWER studies for ITC analyses was assessed by comparing study designs, patient populations, and outcomes definitions of the two studies (Supplementary Table 1). Differences expected to potentially impact the results were adjusted in the analyses where possible.

\section{Inclusion/Exclusion Criteria}

Many of the key inclusion and exclusion criteria were similar; however, there were also some considerable differences between the two studies. First, INO-VATE-ALL enrolled approximately $15 \%$ of patients with Philadelphia chromosome-positive $(\mathrm{Ph}+)$ precursor B cell ALL; in contrast, TOWER included only patients with Ph-negative $(\mathrm{Ph}-)$ precursor B cell ALL. Only $\mathrm{Ph}-$ patients from INO-VATE-ALL were included in the MAIC and STC analyses. Unlike INO-VATE-ALL, patients with high peripheral blasts $(>10,000 / \mu \mathrm{L})$ at baseline were eligible for enrollment in the TOWER study. There was no limit on the number of salvage therapies in TOWER (23\% of patients had three or more), while in INO-VATE-ALL only patients with one or two salvage therapies were enrolled.

\section{Comparator Arm in INO-VATE-ALL and TOWER}

The composition of the comparator arms for both studies was not identical; however, the chemotherapy regimens were sufficiently similar to expect comparable efficacy results. Three different chemotherapy regimens were permitted in the INO-VATE-ALL study, while four different types of chemotherapy regimens were allowed in the TOWER study. The most commonly used SoC regimen in the INO-VATE-ALL study was the combination of fludarabine, highdose cytarabine, and granulocyte colony-stimulating factor (FLAG)-based chemotherapy, whereas the most commonly used SoC regimen in the TOWER study was FLAG chemotherapy with or without an anthracycline.

\section{Outcome Definitions}

The main outcomes of interest for this analysis were remission rate, HSCT rate, OS, and EFS. Although complete response with partial hematologic recovery (CRh) was not reported separately in INO-VATE-ALL, it was included as part of the CRi endpoint. Therefore, for remission rate analyses, the proportion of patients who achieved CR/CRi in INO-VATE-ALL was compared with the proportion of patients who achieved CR/CRi/CRh in TOWER. CR, CRi, and CRh in TOWER had to occur within 12 weeks of the first dose of therapy. Although this restriction was not employed in INO-VATE-ALL, all patients in this study achieved CR/CRi within 3.3 months of the start of treatment. All patients who received a transplant regardless of treatment response or timing were considered in the HSCT rate analysis.

\section{Statistical Analysis}

\section{Network Meta-analysis}

A standard pairwise Bucher method [18] was used to estimate an ITC between InO and Blina, using the relative effect for $\mathrm{InO}$ versus SoC in INO-VATE-ALL and the published relative effects for Blina versus SoC in TOWER derived from intention-to-treat (ITT) comparisons.

\section{MAIC and STC}

For each outcome, likely treatment-effect modifiers were identified from the literature, stratified analyses from both the INO-VATE-ALL and TOWER studies, and clinical experts' input. Table 1 summarizes the treatment-effect modifiers used for the anchored MAIC/STC analyses. Duration of the first remission is a stronger predictor and treatment-effect modifier among patients with only one salvage therapy than in patients in a second or later salvage phase [19]. Therefore, an adjustment in the analyses was made for the proportions of patients in each salvage treatment phase (first, or second or later) combined with the duration of the first remission (less than 12 months or equal to/greater than 12 months). 
Table 1 Treatment-effect modifiers selected for analysis

\begin{tabular}{|c|c|c|c|}
\hline \multirow[t]{2}{*}{ Factor } & \multicolumn{3}{|c|}{$\begin{array}{l}\text { Treatment effect } \\
\text { modifier? }\end{array}$} \\
\hline & $\begin{array}{l}\mathrm{CR} / \\
\mathrm{CRi}\end{array}$ & HSCT & $\begin{array}{l}\text { OS/ } \\
\text { EFS }\end{array}$ \\
\hline Age & Yes & Yes & Yes \\
\hline Philadelphia chromosome status & Yes & No & Yes \\
\hline Prior HSCT & Yes & Yes & Yes \\
\hline Duration of first remission & Yes & No & No \\
\hline $\begin{array}{l}\text { Prior number of salvage } \\
\text { therapies }\end{array}$ & Yes & No & No \\
\hline $\begin{array}{l}\text { Maximum of central/local bone } \\
\text { marrow blasts }\end{array}$ & Yes & No & No \\
\hline Geographical region & No & Yes & Yes \\
\hline
\end{tabular}

$C R$ complete response, $C R i$ complete remission with incomplete hematologic recovery, EFS event-free survival, $H S C T$ hematopoietic stem cell transplantation, $O S$ overall survival

The anchored MAIC technique [19] balances differences in potential treatment-effect modifiers through propensity score re-weighting of patients from INO-VATE-ALL to produce a patient profile matching that of TOWER. Estimates of relative effect (i.e., InO versus SoC in a TOWER-like population) were then derived for outcomes of interest using the re-weighted population. For rates of remission and HSCT, the relative effects of $\mathrm{InO}$ versus SoC in the TOWER-like population were quantified using an odds ratio (OR) and 95\% confidence interval (CI) derived from a weighted logistic regression analysis. For OS and EFS, the relative effect was quantified as a hazard ratio (HR) with a 95\% CI derived from a weighted Cox regression analysis (unstratified). To account for potential violation of the proportional hazard assumption due to differences in short- and long-term performance against SoC, as shown in the published OS and EFS curves in both studies, time-dependent Cox regression [20] and restricted mean survival time (RMST) [21] approaches were also performed to quantify differences in OS and EFS. Data from published OS and EFS Kaplan-Meier curves for the Blina and SoC chemotherapy arms for the ITT population were extracted and digitized, and the Guyot method [22] was used to derive virtual patient-level data to calculate RMST and perform time-dependent analyses on OS in TOWER.

In the anchored STC [15], patient-level data from INO-VATE-ALL were used to create a separate predictive equation for each outcome of interest, which were then used to estimate the relative treatment effect of $\mathrm{InO}$ versus SoC in a TOWER-like population. All treatment-effect modifiers were included in the regression equations. Logistic regression models were used for rates of remission and HSCT and the relative effect was quantified as an OR with a 95\% CI. Results were also presented as differences in rates with an approximate 95\% CI. Cox proportional hazard models were used for OS and EFS, and the relative effect was quantified as an HR with a $95 \%$ CI. As a result of the non-proportional treatment effect over time, time-dependent treatment effects on OS for InO versus SoC were also derived.

Treatment effects for InO versus Blina were derived using the Bucher method, which compared adjusted relative effects derived from anchored MAIC or STC analyses of InO versus SoC in INO-VATE-ALL and published (or obtained using virtual patient-level data) relative effects for Blina versus SoC in TOWER.

\section{RESULTS}

\section{Matching Patient Baseline Characteristics}

Patient baseline characteristics for the ITT population of both studies are presented in Table 2 . Before matching, there were considerable differences between populations in $\mathrm{Ph}$ chromosome status, number of previous salvage therapies, duration of first remission, age, geographic region, and history of HSCT.

Once the weights were applied, all proportions for the set of patient characteristics included in the matching were similar between the two study populations. However, it was not possible to fully account for differences in the number of salvage treatment phases, because the TOWER study placed no limit on the 
Table 2 Patient baseline characteristics

\begin{tabular}{|c|c|c|c|c|}
\hline \multirow[t]{2}{*}{ Patient characteristics } & \multicolumn{2}{|c|}{ INO-VATE-ALL } & \multicolumn{2}{|l|}{ TOWER } \\
\hline & SoC & InO & $\overline{\text { SoC }}$ & Blina \\
\hline$N$ & 162 & 164 & 134 & 271 \\
\hline \multicolumn{5}{|l|}{ Age (years) } \\
\hline Median & 48 & 47 & 37 & 37 \\
\hline Range & $18-79$ & $18-78$ & $18-78$ & $18-80$ \\
\hline$<35, n(\%)$ & $51(31.5)$ & $54(32.9)$ & $60(44.8)$ & $124(45.8)$ \\
\hline$\geq 35, n(\%)$ & $111(68.5)$ & $110(67.1)$ & $74(55.2)$ & $147(54.2)$ \\
\hline \multicolumn{5}{|l|}{ Geographic region, $n(\%)$} \\
\hline Europe & $66(40.7)$ & $61(37.2)$ & $85(63.4)$ & $180(66.4)$ \\
\hline USA or Canada & $79(48.8)$ & $75(45.7)$ & $23(17.2)$ & $41(15.1)$ \\
\hline Rest of world & $17(10.5)$ & $28(17.1)$ & $26(19.4)$ & $50(18.5)$ \\
\hline \multicolumn{5}{|l|}{ Salvage treatment phase (investigator), $n(\%)^{\mathrm{a}}$} \\
\hline First & $102(63.0)$ & $111(67.7)$ & $63(47.0)$ & $104(38.4)$ \\
\hline Second or later & $59(36.4)$ & $51(31.1)$ & $71(53.0)$ & $167(61.6)$ \\
\hline Missing & $1(0.6)$ & $2(1.2)$ & 0 & 0 \\
\hline First remission duration less than 12 months (investigator), $n(\%)^{\mathrm{a}}$ & $106(65.4)$ & $96(58.5)$ & $49(36.6)$ & $109(40.2)$ \\
\hline \multicolumn{5}{|l|}{ Salvage treatment phase by duration of first remission (investigator), $n(\%)^{\mathrm{a}, \mathrm{b}}$} \\
\hline First salvage treatment with duration of first remission $<12$ months & $62(38.5)$ & $63(38.9)$ & $30(22.4)$ & $58(21.4)$ \\
\hline$\geq 2$ salvage treatments with duration of first remission $<12$ months & $43(26.7)$ & $31(19.1)$ & $19(14.2)$ & $51(18.8)$ \\
\hline First salvage treatment with duration of first remission $\geq 12$ months & $40(24.8)$ & $48(29.6)$ & $33(24.6)$ & $46(17.0)$ \\
\hline$\geq 2$ salvage treatments with duration of first remission $\geq 12$ months & $16(10.0)$ & $20(12.4)$ & $52(38.8)$ & $116(42.8)$ \\
\hline \multicolumn{5}{|l|}{ Previous HSCT, $n(\%)^{\mathrm{c}}$} \\
\hline Yes & $32(19.8)$ & $29(17.7)$ & $46(34.3)$ & $94(34.7)$ \\
\hline No & $130(80.2)$ & $135(82.3)$ & $87(64.9)$ & $176(64.9)$ \\
\hline Unknown & 0 & 0 & $1(0.7)$ & $1(0.4)$ \\
\hline \multicolumn{5}{|l|}{ Maximum of central/local bone marrow blasts, $n(\%)$} \\
\hline$<50 \%$ & $48(29.6)$ & $53(32.3)$ & $30(22.4)$ & $69(25.4)$ \\
\hline$\geq 50 \%$ & $113(69.8)$ & $109(66.5)$ & $104(77.6)$ & $201(74.2)$ \\
\hline Missing data & $1(0.6)$ & $2(1.2)$ & 0 & $1(0.4)$ \\
\hline
\end{tabular}


Table 2 continued

\begin{tabular}{llllll}
\hline Patient characteristics & \multicolumn{2}{l}{ INO-VATE-ALL } & \multicolumn{2}{l}{ TOWER } \\
& SoC & InO & SoC & Blina \\
\hline $\mathrm{Ph}+\mathrm{B}$ precursor ALL status, $n(\%)$ & $27(16.7)$ & $22(13.4)$ & 0 & 0 \\
\hline
\end{tabular}

ALL acute lymphoblastic leukemia, Blina blinatumomab, HSCT hematopoietic stem cell transplantation, InO inotuzumab ozogamicin, $\mathrm{Ph}$ Philadelphia chromosome, $\mathrm{So} C$ standard of care

a Salvage treatment phase and duration of first remission per case report form in INO-VATE-ALL and as adjudicated by the investigator in TOWER

b Without considering the patients with a missing salvage treatment phase in INO-VATE-ALL trial $(n=2$ for InO and $n=1$ for SoC)

c Allogeneic HSCT for TOWER trial

number of salvage therapies $(23 \%$ of patients had three or more), whereas the INO-VATE-ALL study enrolled only patients with no more than two salvage therapies. Thus, the matched proportion of patients with two or more salvage treatment phases in INO-VATE-ALL includes only patients with two salvage treatment phases, whereas in TOWER it also includes patients who had three or more. The INO-VATE-ALL sample size after matching (i.e., effective sample size) was reduced by approximately $50 \%$ or more for all outcomes, except HSCT rate (Table 3; Supplementary Table 2).

\section{Indirect Treatment Comparisons}

In comparison to the results obtained using NMA, anchored MAIC and STC results indicated stronger treatment effects for InO relative to Blina for most of the outcomes (Table 3; Supplementary Table 2). As shown in Table 3, the odds of remission were statistically significantly greater with InO when compared with Blina, regardless of the ITC method used (OR [95\% CI] NMA 2.63 [1.35, 5.12]; MAIC 2.81 [1.12, 7.05]; STC $3.91[1.53,9.99])$. When comparing HSCT rates between InO and Blina, results indicated a statistically significantly higher rate among patients who received InO (OR [95\% CI] NMA 3.23 [1.63, 6.40]; MAIC 4.11 [1.85, 9.12]; STC $3.77[1.71,8.35])$. Similarly, when treatment effects were estimated in terms of rate difference, results also indicated statistically significantly higher remission and HSCT rates for InO versus Blina (remission rate difference [95\% CI]
NMA 23.64 [10.10, 37.20]; MAIC 25.12 [6.60, 43.70]; STC 31.44 [13.80, 49.10] and HSCT rate difference [95\% CI] NMA 25.85 [12.50, 39.20]; MAIC 31.03 [15.50, 46.50]; STC 29.33 [13.70, 44.90]).

For EFS, the ITC analyses indicated a favorable trend for InO compared with Blina (Supplementary Table 2; Fig. 1; see TOWER study Fig. 1c [11]). With the MAIC adjustment, results showed statistically significantly higher RMST differences and ratios for InO compared with Blina, suggesting a longer mean EFS for patients who received InO. Results were not statistically significant when the EFS treatment effect was quantified using an HR.

The OS curves appeared to depart from the proportional hazards assumption (Fig. 2), proportionality being required in standard Cox regression analyses. Therefore, the OS HRs should be interpreted with caution (Supplementary Table 2). For OS, overall and time-dependent adjusted HRs (95\% CIs) and RMST difference and ratio from anchored MAIC and STC analyses were consistent and revealed no statistically significant difference in OS between InO and Blina (see TOWER study Fig. 1a [11]).

\section{DISCUSSION}

The aim of this study was to indirectly compare the treatment effects between InO and Blina because of the absence of a head-to-head comparison of these two drugs in adult R/R ALL. Results presented here indicated that remission and HSCT rates were significantly higher for 
Table 3 Efficacy outcomes

\begin{tabular}{|c|c|c|c|c|c|c|c|}
\hline & \multicolumn{2}{|l|}{ ITT } & \multicolumn{2}{|l|}{ Adjusted $^{\mathrm{a}}$} & \multicolumn{3}{|c|}{ Indirect treatment comparisons } \\
\hline & $\begin{array}{l}\text { TOWER } \\
\text { Blina vs. } \\
\text { SoC }\end{array}$ & $\begin{array}{l}\text { INO-VATE- } \\
\text { ALL InO vs. } \\
\text { SoC }\end{array}$ & $\begin{array}{l}\text { INO-VATE- } \\
\text { ALL InO vs. } \\
\text { SoC MAIC }\end{array}$ & $\begin{array}{l}\text { INO-VATE- } \\
\text { ALL InO vs. } \\
\text { SoC STC }\end{array}$ & $\begin{array}{l}\text { InO vs. } \\
\text { Blina } \\
\text { NMA }\end{array}$ & $\begin{array}{l}\text { InO vs. } \\
\text { Blina } \\
\text { MAIC }\end{array}$ & $\begin{array}{l}\text { InO vs. } \\
\text { Blina } \\
\text { STC }\end{array}$ \\
\hline \multicolumn{8}{|l|}{$\mathrm{CR} / \mathrm{CRi}$ rate } \\
\hline $\begin{array}{l}\text { Effective } \\
\text { sample size }\end{array}$ & $271 / 134$ & $164 / 162$ & $70 / 53$ & $138 / 133$ & NA & NA & NA \\
\hline $\begin{array}{c}\text { Odds ratio } \\
(95 \% \mathrm{CI})\end{array}$ & $\begin{array}{c}2.40(1.50 \\
3.81)\end{array}$ & $\begin{array}{c}6.30(3.89 \\
10.21)\end{array}$ & $6.75(3.04,14.95)$ & $\begin{array}{c}9.38(4.14 \\
21.25)\end{array}$ & $\begin{array}{l}2.63 \\
(1.35 \\
5.12)\end{array}$ & $\begin{array}{l}2.81 \\
\quad(1.12 \\
7.05)\end{array}$ & $\begin{array}{l}3.91 \\
(1.53 \\
9.99)\end{array}$ \\
\hline $\begin{array}{l}\text { Remission rate } \\
\text { difference } \\
(95 \% \mathrm{CI})\end{array}$ & $\begin{array}{l}19.28 \\
(9.90 \\
28.67)\end{array}$ & $\begin{array}{l}42.92(33.12 \\
52.71)\end{array}$ & $\begin{array}{l}44.40(28.42 \\
60.38)\end{array}$ & $\begin{array}{c}50.72(35.74 \\
65.70)^{\mathrm{b}}\end{array}$ & $\begin{array}{l}23.64 \\
\quad(10.10 \\
37.20)\end{array}$ & $\begin{array}{l}25.12 \\
\quad(6.60, \\
43.70)\end{array}$ & $\begin{array}{l}31.44 \\
(13.80, \\
49.10)\end{array}$ \\
\hline \multicolumn{8}{|l|}{ HSCT rate } \\
\hline $\begin{array}{l}\text { Effective } \\
\text { sample size }\end{array}$ & $271 / 134$ & $164 / 162$ & $100 / 94$ & $164 / 162$ & NA & NA & NA \\
\hline $\begin{array}{c}\text { Odds ratio } \\
(95 \% \mathrm{CI})\end{array}$ & $\begin{array}{c}1.01(0.62 \\
1.63)\end{array}$ & $\begin{array}{c}3.25(2.01 \\
5.26)\end{array}$ & $4.15(2.20,7.85)$ & $3.81(2.03,7.18)$ & $\begin{array}{c}3.23 \\
(1.63 \\
6.40)\end{array}$ & $\begin{array}{l}4.11 \\
\quad(1.85 \\
9.12)\end{array}$ & $\begin{array}{l}3.77 \\
\quad(1.71 \\
8.35)\end{array}$ \\
\hline $\begin{array}{l}\text { HSCT rate } \\
\text { difference } \\
(95 \% \mathrm{CI})\end{array}$ & $\begin{array}{l}0.10 \\
\quad(-8.72 \\
8.93)\end{array}$ & $\begin{array}{c}25.95(15.98 \\
35.92)\end{array}$ & $\begin{array}{l}31.13(18.40 \\
43.85)\end{array}$ & $\begin{array}{c}29.43(16.59 \\
42.27)^{\mathrm{b}}\end{array}$ & $\begin{array}{l}25.85 \\
\quad(12.50 \\
39.20)\end{array}$ & $\begin{array}{c}31.03 \\
(15.50 \\
46.50)\end{array}$ & $\begin{array}{l}29.33 \\
\quad(13.70, \\
44.90)\end{array}$ \\
\hline
\end{tabular}

Remission/HSCT rates were estimated from logistic model, which can lead to over/underestimation of the true uncertainty Standard errors for the estimated remission/HSCT rates were approximated using 95\% CI for proportion derived from logistic regression model (estimates not shown but used to calculate uncertainty for the difference in remission/HSCT rates) Blina blinatumomab, CI confidence interval, $C R$ complete remission, CRi complete remission with incomplete hematologic recovery, $H S C T$ hematopoietic stem cell transplantation, In $O$ inotuzumab ozogamicin, ITT intention-to-treat (randomized controlled study population), MAIC matching-adjusted indirect comparison, $N A$ not applicable, $N M A$ network metaanalysis, $S o C$ standard of care, $S T C$ simulated treatment comparison

a The adjusted MAIC and STC analyses were performed on the basis of the treatment-effect modifiers list, which is outcome-specific and presented in Table 1

b Limitations for STC method presented as remission/HSCT rate differences

InO compared to Blina regardless of the ITC method applied. After adjustment using anchored MAIC and STC analyses, the estimated treatment effect became stronger with InO than when using an unadjusted naïve analysis. Results from the anchored MAIC and STC approaches were generally consistent. Results also suggested longer EFS with InO than Blina. Although a statistically significant difference was not observed when EFS was quantified using HRs, RMST differences and ratios from the MAIC analysis significantly favored InO over Blina.

Results from both the overall and time-dependent analyses indicated that there was no significant difference between $\mathrm{InO}$ and Blina in OS. Given the limited follow-up data from TOWER (due to the study meeting its early 
a

Product-Limit Survival Estimates

With Number of Subjects at Risk
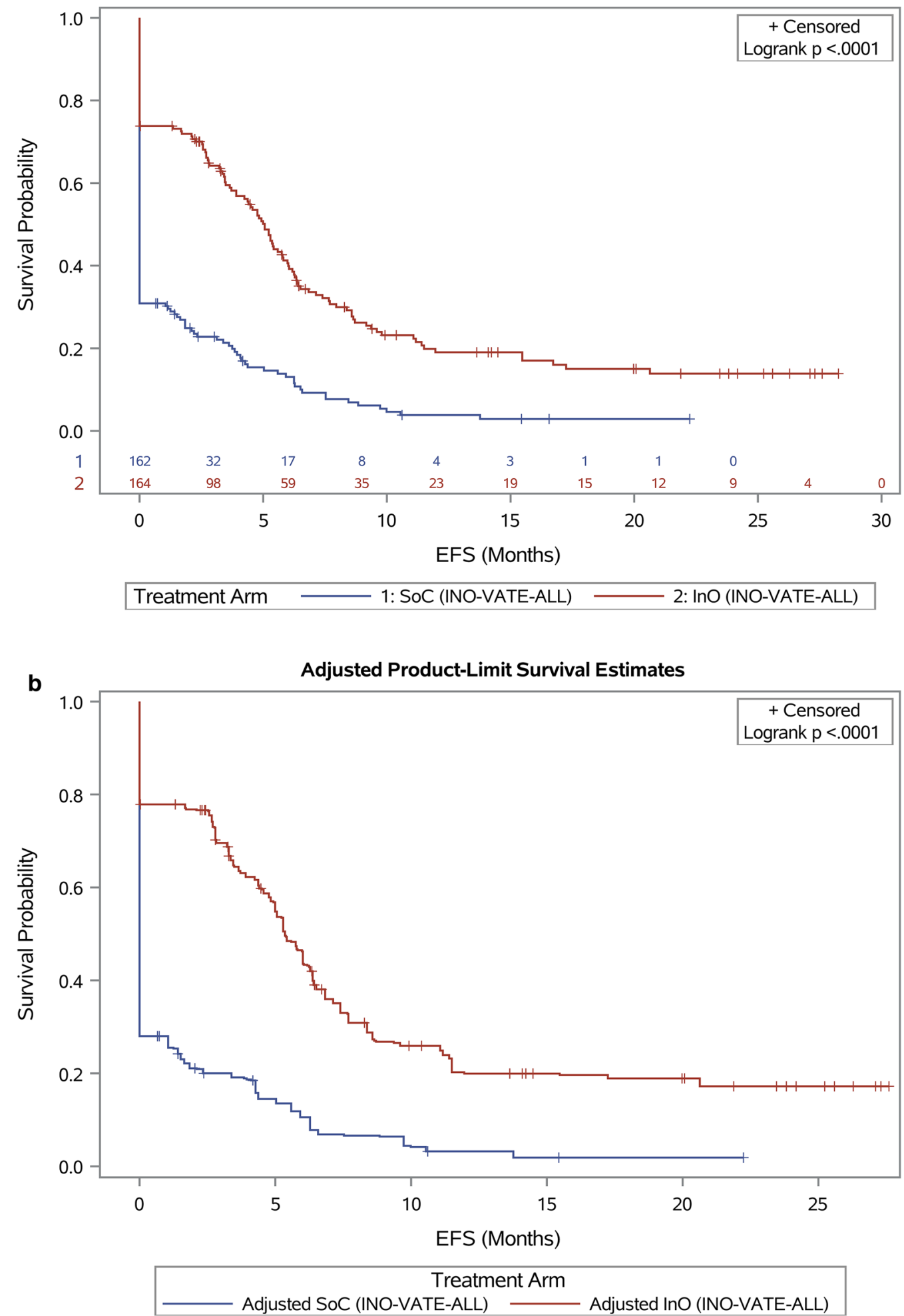

Fig. 1 Event-free survival Kaplan-Meier curves before matching (a) and after matching (b). a INO-VATE-ALL before matching. b INO-VATE-ALL after matching. EFS event-free survival, InO inotuzumab ozogamicin, $S o C$ standard of care 

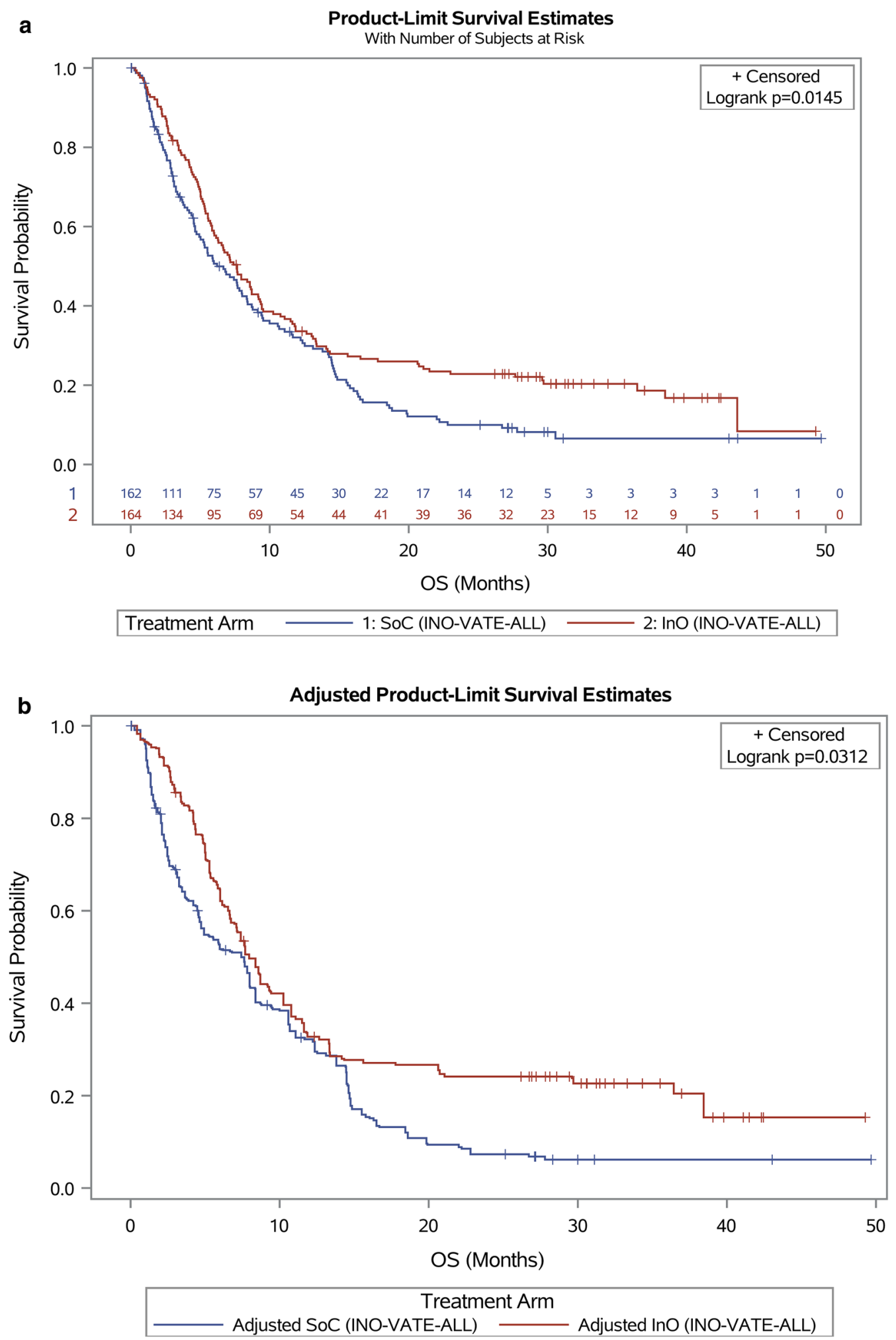

Fig. 2 Overall survival Kaplan-Meier curve before matching (a) and after matching (b). a INO-VATE-ALL before matching. b INO-VATE-ALL after matching. In $O$ inotuzumab ozogamicin, $O S$ overall survival, $S o C$ standard of care 
stopping criteria at month 20), relative effects could not be reliably estimated after 15 months of follow-up; this should be considered when interpreting the results of this analysis. In INOVATE-ALL, the separation in OS curves between InO and SoC accelerated at 15 months, indicating longer-term survival benefit, which is consistent with the higher HSCT rates that were observed among patients in the InO arm. For the TOWER study, the OS curves for Blina and SoC converged at about this same time point for the ITT population, which is consistent with the identical HSCT rates observed among patients in the Blina and SoC arms. In light of these observations, it is possible that the findings from the ITC may underestimate the relative OS for InO.

Recently, anchored MAIC analyses comparing Blina to InO were conducted and published by Song et al. 2019 for OS and CR rate [23]. This study found no difference in CR rates; furthermore, mean restricted survival for Blina was 1.6 months (95\% CI $[0.1,3.2] ; p<0.05)$ longer than for InO when the authors applied the RMST method using only 12 months of OS data. However, when the entire follow-up period was considered, no statistically significant RMST difference in OS was found between $\mathrm{InO}$ and Blina. Authors used patient-level data from the TOWER study and published data from the INO-VATE-ALL study. Since the TOWER study enrolled only $\mathrm{Ph}$ - precursor B cell ALL patients, it was not possible to adjust for this in the analyses conducted by Song et al. and therefore reported results could potentially be biased in favor of Blina.

Alternatively, authors could fully adjust for the number of salvage therapies by excluding patients who had received three or more therapies; this was not possible in our analyses. The HSCT or CRi/CRh remission rates were not compared, despite remission rate being the primary endpoint in both studies. As discussed by Song et al., the definitions of CRi/CRh between the TOWER and INO-VATE-ALL studies were not identical. However, all our ITC analyses were anchored; therefore, some differences in definition are unlikely to impact the relative effects within the study or change the results for the derived treatment effects of InO versus
Blina. The CRi/CRh rates are important to include as they also enable patients to proceed to HSCT and benefit from long-term survival and potential cure.

The evidence-based healthcare decisionmaking for clinical treatment guidelines and reimbursement policies requires comparisons of all relevant competing interventions. Direct comparative studies are rarely available in the time period immediately following approval of a treatment since the primary goal of clinical development is to meet regulatory requirements $[14,15]$. In the absence of randomized controlled trials involving a direct comparison of all treatments of interest, ITCs provide useful evidence for judiciously selecting the best choice(s) of treatment [24].

The indirect comparisons made here are subject to limitations. NMA and anchored MAIC and STC analyses rely on a common comparator arm. The SoC intensive chemotherapy regimens used as the comparator arms for INO-VATE-ALL and TOWER were not identical. Since there are no randomized clinical studies that compared those regimens head-tohead, it is possible that some SoC regimens are slightly more efficacious than others; however, it is important to note that in both studies, the remission rate with SoC was low, as is consistent with historical data.

In our analyses, the SoC regimens were considered similar in terms of efficacy. MAIC and STC analyses depend on all treatment-effect modifiers being adjusted for in the analyses. Although we adjusted for most of the treatment-effect modifiers identified in the analyses, some were either not or only partially adjusted, such as the number of prior salvage therapies and high peripheral blasts. Thus, treatment effect for InO over Blina may have been overestimated. The effective sample size in MAIC analyses was reduced by $50 \%$ or more for most of the outcomes evaluated, which led to wider 95\% CIs and an increased uncertainty in the results.

\section{CONCLUSIONS}

The results of this adjusted ITC showed a statistically significant advantage for InO 
compared with Blina on improving rates of remission and HSCT. Further head-to-head randomized controlled studies of InO versus Blina are needed to confirm the findings presented in this manuscript.

\section{ACKNOWLEDGEMENTS}

The authors would like to thank all study participants for their involvement in the studies used to derive this work.

Funding. This study, the development of this article, the article processing charges, and the fee for Open Access publication were all funded by Pfizer Inc, New York, NY. All authors had full access to all of the data in this study and take complete responsibility for the integrity of the data and accuracy of the data analysis.

Medical Writing, Editorial, and Other Assistance. The authors would like to thank the following contributions: Kendra Hughes, PharmD, (Evidera, Raleigh NC) for medical writing, editing, and data preparation, funded by Pfizer. Janet Dooley (Evidera, Waltham, MA) provided editorial and submission support, funded by Pfizer. The authors would also like to thank Tao Wang (Pfizer) for his thoughtful contributions to the manuscript.

Authorship. All authors meet the International Committee of Medical Journal Editors (ICMJE) criteria for authorship for this article, take responsibility for the integrity of the work as a whole, and have provided their approval for this version to be published.

Authorship Contributions. Irina Proskorovsky made a substantial contribution to the research design; analysis and interpretation of data; revised draft versions of the manuscript for important intellectual content; and provided final approval of the version to be published. Yun $\mathrm{Su}$ contributed to the conceptualization, design, analysis, and interpretation of the study results; critically revised draft versions of the manuscript for important intellectual content; and gave final approval of the version to be published. Kyle Fahrbach made a substantial contribution to the research design, analysis and interpretation of data; made revisions to draft versions of the manuscript; and gave final approval of the version to be published. Erik Vandendries contributed to the study design (original B1931022 design), interpretation of the data, revised draft versions of the manuscript for intellectual content, and approved the final manuscript. Véronique Pagé contributed to the study design, analysis, and interpretation of data; revised draft versions of the manuscript for important intellectual content; and gave final approval of the version to be published. Uchenna Onyekwere contributed to the analysis and interpretation of the data, made revisions to draft versions of the manuscript, and gave final approval of the version to be published. Yunyang Wang contributed to the analysis and interpretation of data; reviewed the manuscript for intellectual content; and approved the final version of the manuscript. Joseph C. Cappelleri contributed to the analysis and interpretation of data; made revisions of draft versions of the manuscript and gave important intellectual context; and gave final approval of the manuscript. Matthias Stelljes contributed to the study design, analysis, and interpretation of the data; revised draft versions of the manuscript; and gave final approval.

Disclosures. Joseph C. Cappelleri is an employee and shareholder of Pfizer Inc, which funded this study. Erik Vandendries is an employee and shareholder of Pfizer Inc. Yun Su was an employee and shareholder of Pfizer Inc during conduct of this study; Yun Su is currently employed by Eisai Inc. Irina Proskorovsky is a salaried employee of Evidera and is forbidden from accepting remuneration of any kind from clients of Evidera. Véronique Pagé is a salaried employee of Evidera and is forbidden from accepting remuneration of any kind from clients of Evidera. Yunyang Wang is a salaried employee of Evidera and is forbidden from accepting remuneration of any kind from clients of Evidera. Kyle Fahrbach is a salaried employee of Evidera and is forbidden from 
accepting remuneration of any kind from clients of Evidera. Uchenna Onyekwere was employed by Evidera during the conduct of the study and is currently employed by IBM Watson Health. Evidera received funding from Pfizer to conduct this study and develop associated publications. Matthias Stelljes reports research support from Pfizer during the conduct of the study, and other consulting fees and honoraria from Pfizer, Amgen, and Jazz Pharmaceuticals.

Compliance with Ethics Guidelines. No institutional board review was required for this study as it was based on a post hoc analysis of previously published data from the INO-VATEALL and TOWER trials. These previous studies involved human participants and were conducted in accordance with the ethical standards of the institutional and/or national research committees of each study's investigative sites, and with the 1964 Helsinki declaration and its later amendments, or comparable ethical standards. Informed consent was obtained from all individual participants included in these previous trials.

Data Availability. Data sharing is not applicable to this article as no datasets were generated or analyzed during the current study.

Open Access. This article is distributed under the terms of the Creative Commons Attribution-NonCommercial 4.0 International License (http://creativecommons.org/licenses/ by-nc/4.0/), which permits any noncommercial use, distribution, and reproduction in any medium, provided you give appropriate credit to the original author(s) and the source, provide a link to the Creative Commons license, and indicate if changes were made.

\section{REFERENCES}

1. National Comprehensive Cancer Network (NCCN). NCCN Clinical Practice Guidelines in Oncology (NCCN Guidelines ${ }^{\circledR}$ ). Acute lymphoblastic leukemia. Version 1.2018. Plymouth Meeting, PA: National Comprehensive Cancer Network; 2018. https://www.nccn.org/professionals/physician_gls/ default.aspx. Accessed Mar 122018.

2. Terwilliger T, Abdul-Hay M. Acute lymphoblastic leukemia: a comprehensive review and 2017 update. Blood Cancer J. 2017;7(6):e577. https://doi. org/10.1038/bcj.2017.53.

3. $\quad$ PDQ $^{\circledR}$ Adult Treatment Editorial Board. PDQ ${ }^{\circledR}$ Adult acute lymphoblastic leukemia treatment. Healthcare professional version. PMID 26389171 Bethesda, MD: National Cancer Institute; 2019 (updated 8 February 2019). https://www.cancer. gov/types/leukemia/hp/adult-all-treatment-pdq. Accessed Mar 42019.

4. Siegel RL, Miller KD, Jemal A. Cancer statistics, 2018. CA Cancer J Clin. 2018;68(1):7-30. https:// doi.org/10.3322/caac. 21442 .

5. Tricoli JV, Blair DG, Anders CK, et al. Biologic and clinical characteristics of adolescent and young adult cancers: acute lymphoblastic leukemia, colorectal cancer, breast cancer, melanoma, and sarcoma. Cancer. 2016;122(7):1017-28. https://doi. org/10.1002/cncr.29871.

6. Wei G, Wang J, Huang H, Zhao Y. Novel immunotherapies for adult patients with B-lineage acute lymphoblastic leukemia. J Hematol Oncol. 2017;10(1):150. https://doi.org/10.1186/s13045-0170516-X.

7. Gokbuget N, Stanze D, Beck J, et al. Outcome of relapsed adult lymphoblastic leukemia depends on response to salvage chemotherapy, prognostic factors, and performance of stem cell transplantation. Blood. 2012;120(10):2032-41. https://doi.org/10. 1182/blood-2011-12-399287.

8. Tavernier E, Boiron JM, Huguet F, et al. Outcome of treatment after first relapse in adults with acute lymphoblastic leukemia initially treated by the LALA-94 trial. Leukemia. 2007;21(9):1907-14. https://doi.org/10.1038/sj.leu.2404824.

9. BLINCYTO ${ }^{\circledR}$ (blinatumomab) Package Insert. Revised 5/2018. Initial US approval: 2014. Thousand Oaks: Amgen, Inc; 2018.

10. BESPONSA ${ }^{\circledR}$ (inotuzumab ozogamicin) Package Insert. Revised: 3/2018. Initial US approval: 2017. Philadelphia: Wyeth Pharmaceuticals, a subsidiary of Pfizer Inc; 2018.

11. Kantarjian H, Stein A, Gokbuget N, et al. Blinatumomab versus chemotherapy for advanced acute lymphoblastic leukemia. N Engl J Med. 2017;376(9):836-47. https://doi.org/10.1056/ NEJMoa1609783. 
12. Kantarjian HM, DeAngelo DJ, Stelljes M, et al. Inotuzumab ozogamicin versus standard therapy for acute lymphoblastic leukemia. N Engl J Med. 2016;375(8):740-53. https://doi.org/10.1056/NEJM oa1509277.

13. Dias S, Ades AE, Welton NJ, Jansen JP, Sutton AJ. Network meta-analysis for decision-making. Chichester: Wiley; 2018.

14. Ishak KJ, Proskorovsky I, Benedict A. Simulation and matching-based approaches for indirect comparison of treatments. Pharmacoeconomics. 2015;33(6):537-49. https://doi.org/10.1007/s4027 3-015-0271-1.

15. Caro JJ, Ishak KJ. No head-to-head trial? Simulate the missing arms. Pharmacoeconomics. 2010;28(10):957-67. https://doi.org/10.2165/ 11537420-000000000-00000.

16. Phillippo DM, Ades AE, Dias S, et al. NICE DSU Technical Support Document 18: methods for population-adjusted indirect comparisons in submissions to NICE. Report by the Decision Support Unit. 2016. http://www.nicedsu.org.uk. Accessed Dec 72018.

17. Dombret H, Topp MS, Schuh A, et al. Blinatumomab vs. SOC chemotherapy in first salvage compared with second or greater salvage in a phase 3 study. Oral presentation, Abstract S478. 22nd Congress of the European Hematology Association; June 22-25, 2017; Madrid, Spain; 2017.

18. Bucher HC, Guyatt GH, Griffith LE, Walter SD. The results of direct and indirect treatment comparisons in meta-analysis of randomized controlled trials. J Clin Epidemiol. 1997;50(6):683-91.
19. Signorovitch JE, Sikirica V, Erder $\mathrm{MH}$, et al. Matching-adjusted indirect comparisons: a new tool for timely comparative effectiveness research. Value Health. 2012;15(6):940-7. https://doi.org/10. 1016/j.jval.2012.05.004.

20. Kleinbaum DG, Klein M. Extension of the cox proportional hazards model for time-dependent variables. Survival analysis: a self-learning text. 3rd ed. New York: Springer; 2012. p. 241-88.

21. Pak K, Uno H, Kim DH, et al. Interpretability of cancer clinical trial results using restricted mean survival time as an alternative to the hazard ratio. JAMA Oncol. 2017;3(12):1692-6. https://doi.org/ 10.1001/jamaoncol.2017.2797.

22. Guyot P, Ades AE, Ouwens MJ, Welton NJ. Enhanced secondary analysis of survival data: reconstructing the data from published Kaplan-Meier survival curves. BMC Med Res Methodol. 2012;12:9. https://doi.org/ 10.1186/1471-2288-12-9.

23. Song J, Ma Q, Gao W, et al. Matching-adjusted indirect comparison of blinatumomab vs. inotuzumab ozogamicin for adults with relapsed/refractory acute lymphoblastic leukemia. Adv Ther. 2019;36(4):950-61. https://doi.org/10.1007/s1232 5-019-0873-7.

24. Jansen JP, Fleurence R, Devine B, et al. Interpreting indirect treatment comparisons and network metaanalysis for health-care decision making: report of the ISPOR Task Force on Indirect Treatment Comparisons Good Research Practices: part 1. Value Health. 2011;14(4):417-28. https://doi.org/10. 1016/j.jval.2011.04.002. 